\title{
Vector Meson Polarization at the Tevatron
}

\author{
James Russ ${ }^{* \dagger}$ \\ Carnegie Mellon University, Pittsburgh, PA USA \\ E-mail: russ@cmphys.phys.cmu.edu
}

Results on Vector Meson Polarization at high $p_{T}$ in $p \bar{p}$ collisions at $1.96 \mathrm{TeV}$ from CDF and D0 are presented. For CDF both $c \bar{c}$ and $b \bar{b}$ states show a tendency toward longitudinal polarization at high $p_{T}$ in the s-channel helicity frame. This disagrees with predictions from NRQCD and agrees with expectations from models with multiple gluon interactions. The D0 measurement of $\Upsilon(1 S)$ polarization tends to go from longitudinal to transverse polarization as $p_{T}(\Upsilon(1 S))$ increases, in disagreement with CDF results.

12th International Conference on B-Physics at Hadron Machines - BEAUTY 2009

September 07 - 112009

Heidelberg, Germany

\footnotetext{
* Speaker.

$\dagger$ on behalf of the CDF and D0 Collaborations
} 


\section{Introduction}

The mechanisms for producing heavy vector mesons, i.e., bound $Q \bar{Q}$ systems, in high energy hadronic collisions remain obscure, despite two decades of intense theoretical and experimental work. The fundamental problem of changing states from an initial colored vector gluon to a colorless $Q \bar{Q}$ hadron has been approached by several modelling schemes. After the initial Tevatron Run I observations of a production cross section that was much larger than the favored color-singlet interaction (CSM) predicted, new approaches emphasizing color-octet contributions (NRQCD) were introduced [1]. It was quickly recognized that the octet mechanism was connected to transverse polarization of the vector meson [2]. Subsequently, attempts to re-invigorate the CSM mechanism by adding gluonic interactions have been made. [6], [7] They have had varying degrees of success, but none is completely satisfactory. The present theoretical situation has been summarized in a recent review article [3]. In all the approaches the spin-dependent coupling in the interaction at high $p_{T}$ produces characteristic polarization effects in the vector meson decays. Therefore, a significant effort has been made at the Tevatron to measure vector meson polarizations at high $p_{T}$ to confront the model predictions with data. I report in this paper on CDF and D0 results for $\mathrm{J} / \psi, \psi(2 S)$, and $\Upsilon(1 S)$ polarization measurements and compare to recent theoretical predictions.

\section{Polarization Measurements at Colliders}

Vector meson polarization is determined from decays $V \rightarrow \mu^{+} \mu^{-}$, using the angular distribution of the final state leptons as determined in the rest frame of the vector meson. The polarization parameter $\alpha$ determines the angular distribution:

$$
\frac{d N}{d(\cos \theta)} \propto 1+\alpha \cos ^{2} \theta
$$

Transverse polarization (T) corresponds to $\alpha=+1$; longitudinal polarization (L) has $\alpha=-1$. A fundamental question, though, is what determines the axis for the polar angle measurement? Traditionally, collider experiments have used the s-channel helicity frame, in which the polar axis is the negative of the boost direction that takes the vector meson from the lab frame to its rest frame. However, fixed target experiments use the Collins-Soper frame [4], for which the axis is the bisector of the angle between the beam and target momenta viewed in the vector meson rest frame. It's not clear that one can ignore the parton transverse momenta in high energy collisions with $p_{T} \sim 25 \mathrm{GeV} / \mathrm{c}$, but most collider groups now are reanalyzing their data in the Collins-Soper frame as well as the s-channel helicity frame. All results in this paper refer to the latter axis.

The same analysis method applies to polarization studies for $\mathrm{J} / \psi, \psi(2 S)$ and $\Upsilon$ mesons. Results will be summarized on the former two from CDF and the latter family from D0 and CDF. Prospects for future Tevatron results will also be given.

\section{CDF Measurement of $\mathbf{J} / \psi$ and $\psi(2 S)$ Polarization}

The CDF collaboration has published results on the $c \bar{c}$ bound states $\mathrm{J} / \psi$ and $\psi(2 S)$. These two states can be produced in three ways: directly; as decays from $c \bar{c}$ excited states including the $\chi_{c}$ 
families; and as decays from b-hadrons. The first two types of decays are very hard to separate at the Tevatron. They are lumped together as prompt events, meaning that the dimuon vertex is part of the primary interaction vertex. In contrast, decay events refer to vector mesons whose vertex position indicates that they are heavy flavor decay products. The analysis separates the polarization measurement for these two classes both for $\mathrm{J} / \psi$ and $\psi(2 S)$ states.

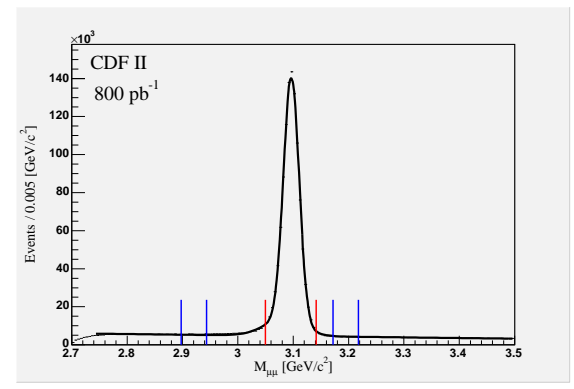

Figure 1: Dimuon Mass

The analysis is done in bins of vector meson transverse momentum. For each $p_{T}$ bin, the dimuon mass distribution is fitted to determine signal and background regions. Prompt and decay events are separated by a selection on the sum of the squared impact parameter significance of the two muons with respect to the primary vertex. There is a small contamination $(\leq 5 \%)$ of decay events in the prompt sample. The exact contamination fraction for each bin is determined from the data by a proper time distribution study.

The mass distribution for a typical prompt $J / \psi$ sample is shown in Fig. 1. Events in the signal and sideband regions, denoted by the vertical markers, are analyzed to produce the muon angular distribution.

Apparatus acceptance, trigger cuts, and reconstruction efficiency all modify the $\mathrm{T}$ and $\mathrm{L}$ theoretical distributions. In the CDF analysis, Monte Carlo simulation is used to transform fully polarized $\mathrm{T}$ and $\mathrm{L}$ vector meson decay samples into pseudo-events that are analyzed by the standard CDF production code. Dead detector channels, additional material, and all trigger conditions are included in the simulation. After production, the $\mathrm{T}$ and $\mathrm{L}$ event samples represent what a fully polarized sample of vector mesons would look like in CDF. These are termed templates. Because any finite polarization is a linear combination of $\mathrm{T}$ and $\mathrm{L}$ samples with a specific relative weight, we make a fitter that compares the measured angular distributions for the signal and sideband regions to a linear combination of the $\mathrm{T}$ and $\mathrm{L}$ templates. The fit procedure does a $\chi^{2}$ fit simultaneously to the background and signal. The results of the fit for $9 \leq p_{T}(\mathrm{~J} / \psi) \leq 12 \mathrm{GeV} / \mathrm{c}$ are shown in Fig. 2 . All the features of the fitter can be seen here. In the left plot, the fitted background histogram is compared to the initial sideband estimate (points). Very little difference is seen, indicating that the sideband angular distributions are good estimators of the background angular distribution under the $\mathrm{J} / \psi$ peak. The background angular distribution is definitely not flat, indicating that good background control is important for polarization analysis. The right plot is the physics result, showing the angular distribution for the signal (points) and the polarization-weighted combination of the $\mathrm{T}$ and $\mathrm{L}$ templates (histogram). The fit quality is excellent at all angles, even at the edges where apparatus acceptance effects are huge.

The polarization for the $\mathrm{J} / \psi$ and $\psi(2 S)$ is shown in Fig. 3. The $\mathrm{J} / \psi$ results show a smooth trend toward $\mathrm{L}$ polarization at higher $p_{T}$. The $\psi(2 S)$ polarization is also negative at large $p_{T}$, but the poor statistical precision limits the conclusions that can be drawn from this state. The $\psi(2 S)$ prompt production cross section is only $3 \%$ of that of the $\mathrm{J} / \psi$ at Tevatron energies. There 

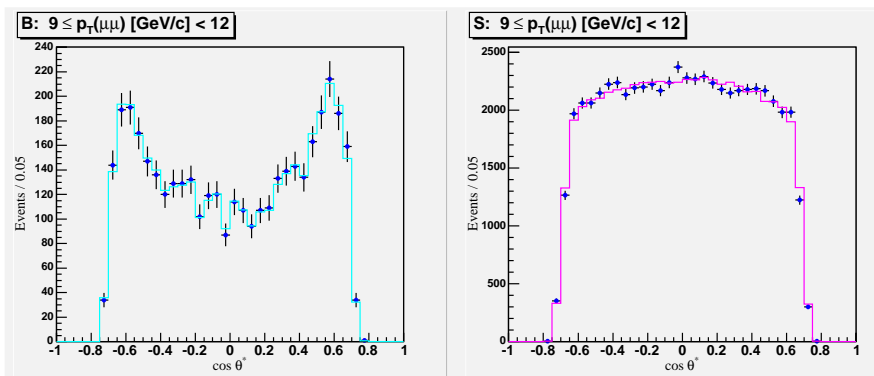

Figure 2: Dimuon angular distribution for data (points) and fit (histogram) . Left: background; Right: Signal

is less feed-down contamination from higher $c \bar{c}$ states in this measurement, because there is no evidence for direct production of the $\mathrm{J} / \psi$ or $\chi$ states above the $\psi(2 S)$ in Tevatron data. NRQCD predictions from Braaten and Lee [5] which include feed-down effects from higher states, do not agree with the trend of the CDF measurement. The multigluon calculations discussed in Ref. 1 predict longitudinal polarization for direct production. They have not yet attempted to include feed-down effects.
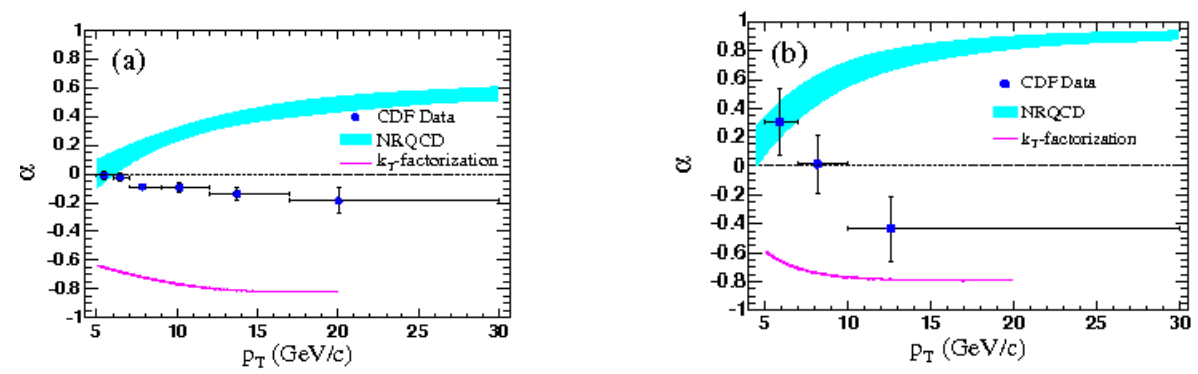

Figure 3: (a) $\mathrm{J} / \psi$ polarization vs. $p_{T}(J / \psi)$; (b) $\psi(2 S)$ polarization vs. $p_{T}(\psi(2 S)$. The NRQCD prediction is from Ref. [5]. The $k_{T}$ factorization prediction is from Ref. [6].

\section{4. $\Upsilon$ Polarization}

There are some suggestions that NRQCD might not work well for charm quark systems because the mass is too low. There is no such question about the $\Upsilon$ family of $b \bar{b}$ bound states, especially for $p_{T}>2 m_{\Upsilon}$ where perturbative effects should dominate. In contrast to the charm states, the $\Upsilon$ family has no decay production channels. There is still feed-down from higher $b \bar{b}$ states, especially for the $\Upsilon(1 S)$ from $\Upsilon(n S)$ and $\chi_{b 1}$ states.

The $\Upsilon(n S)$ family is clearly seen in Tevatron dimuon mass spectra from CDF, as shown in Fig. 4(a). The background level is clearly higher than in the $J / \psi$ case. In the CDF analysis the sideband background for the $\Upsilon(1 S)$ state is estimated from the region between the $1 \mathrm{~S}$ and $2 \mathrm{~S}$ states. $\mathrm{CDF}$ uses the same analysis technique for the $\Upsilon(1 S)$ polarization as is used for the $\mathrm{J} / \psi$. As can be seen from Fig. 4(a), that is not possible for D0 because of poorer mass resolution. The D0 
analysis procedure first separates events into the $\cos \theta^{*}$ distribution, then fits the background and overlapping $\Upsilon(n S)$ signals, using an arbitrary polynomial function for background and a multiGaussian signal function. D0 has tried fitting the signal function both to Monte Carlo and to datadriven shapes. They report that both results give similar signal yields in each angle bin. In contrast to the CDF template method, the D0 procedure for finding the polarization is to first produce an unpolarized Monte Carlo simulation which incorporates trigger efficiency acceptance function. They weight each bin of the unpolarized angular distribution by the probability of getting events in that bin if the polarization parameter were $\alpha$. They apply further weights to match Monte Carlo $p_{T}$ and p distributions to data. The D0 results for $\Upsilon(1 S)$ and $\Upsilon(2 S)$ are published in Ref. [9].
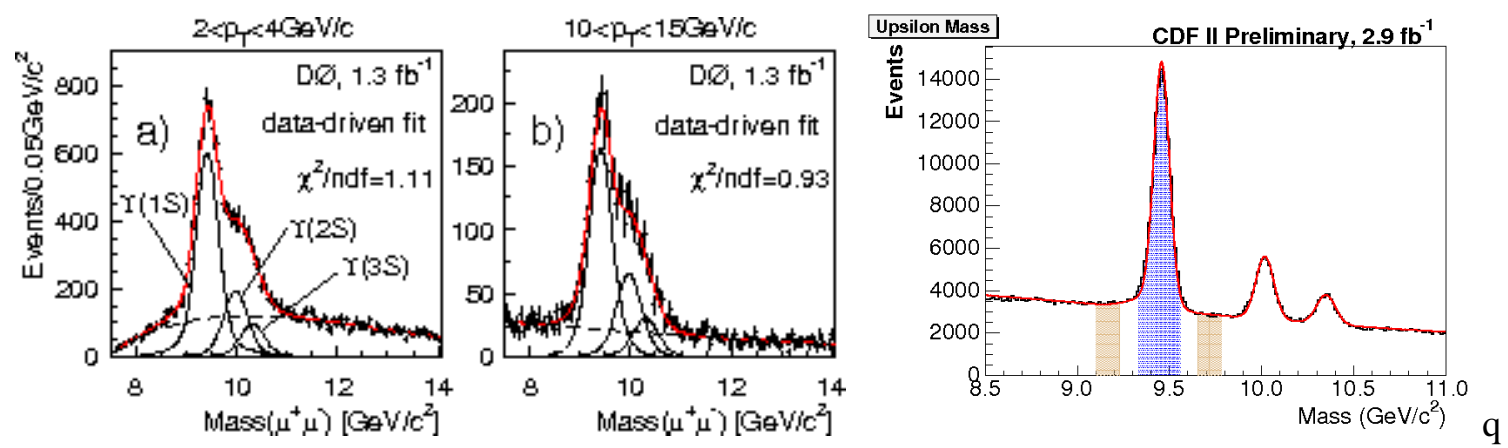

Figure 4: Left: D0 dimuon mass distribution in two dimuon $p_{T}$ bins for rest frame muon angular interval $\left(.4<\left|\cos \theta^{*}\right|<.5\right)$; Right: CDF dimuon mass distribution showing $\Upsilon(1 S)$ signal and sideband regions.

CDF has a new measurement of the $\Upsilon(1 S)$ polarization. [10] It uses the same template method and sideband subtraction procedure that was described above for the $J / \psi$ case. CDF mass and momentum resolution are good enough that there are no significant bin-smearing effects that would cause events to migrate between $p_{T}(\Upsilon(1 S))$ bins or decay angle bins. In this case, too, the sideband background angular distribution is refit to determine the background angular distribution in the signal region. There is good agreement between the two distributions, like in the $\mathrm{J} / \psi$ case. Also in the $\Upsilon(1 S)$ case the background has a great deal of angular structure that varies significantly with dimuon mass and $p_{T}$. The polarization results for D0 and CDF are shown in Fig. 5. CDF Run II polarization agrees well with the CDF Run I result at lower $p_{T}$ and shows a trend toward longitudinal polarization at larger $p_{T}$. The $\mathrm{D} 0$ result for the $1 \mathrm{~S}$ polarization shown in the same figure has considerable $p_{T}$ structure and tends toward T polarization at the largest $p_{T}$, in accord with NRQCD. The two results do not agree at all.

\section{Summary and Future Plans}

The CDF results for all vector meson states studied are consistent with a trend toward longitudinal polarization at high $p_{T}$ in the s-channel helicity frame. This is not consistent with NRQCD but follows the trends predicted in multi-gluon interactions. The CDF data show that the background under both $\mathrm{J} / \psi$ and $\Upsilon(n S)$ states have significant angular structure. How much of that fraction is Drell-Yan production as compared to random dimuon comibnations is an interesting question that will be pursued further at CDF in the future. The multi-gluon models suggest that the $\Upsilon$ states should have additional gluonic activity in their phase space neighborhood. This is an aspect of 


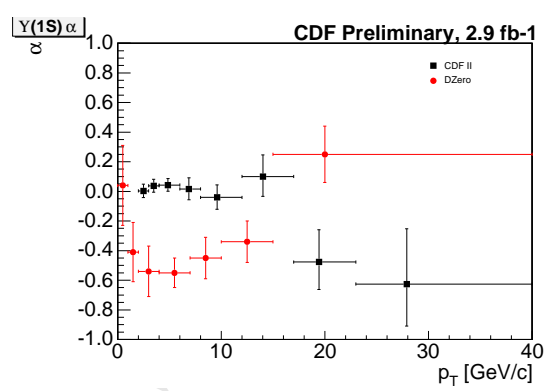

Figure 5: $\Upsilon(1 S)$ polarization vs. $p_{T}(\Upsilon(1 S))$ as measured by CDF and D0.

production that can be studied further but requires collaboration between theory and experiment to determine what sorts of activity should be expected and how it would show up in the detectors.

The CDF group is also analyzing the $\Upsilon(2 S)$ and $\Upsilon(3 S)$ polarization. They are also preparing to redo all the polarization measurements using the Collins-Soper frame. The CDF results presented here will be updated, using a data set twice as large as that presented here for the $\Upsilon(1 S)$ study. D0 expects to report on $\mathrm{J} / \psi$ polarization soon.

\section{References}

[1] G. T. Bodwin, E. Braaten, and G. P. Lepage,Rigorous QCD analysis of inclusive annihilation and production of heavy quarkonium, Phys. Rev. D 51 (1125) 1995; Erratum, ibid., Phys. Rev. D 55 (5853) 1997; E. Braaten and S. Fleming,Color octet fragmentation and the psi-prime surplus at the Tevatron, Phys. Rev. Lett. 74 (3327) 1995.

[2] P. Cho and M. Wise, Spin symmetry predictions for heavy quarkonia alignment, Phys. Lett. B346 (129) 1995; M. Beneke and I. Z. Rothstein, Hadroproduction of quarkonia in fixed target experiments, Phys. Lett. B 372 (157) 1996; Erratum, ibid., Phys. Lett. B 389 (769) 1996;

[3] J.P. Lansberg,On the mechanisms of heavy-quarkonium hadroproduction,Eur. Phys. J. C 61 (693) 2009.

[4] J. Collins and D. Soper, Angular Distribution of Dileptons in High-Energy Hadron Collisions, Phys. Rev. D 16 ( 2219) 1977.

[5] E. Braaten, B.A.Kniehl, and J. Lee, Polarization of prompt J / psi at the Tevatron, Phys. Rev. D 62 (094005) 2000.

[6] S.P.Baranov,Highlights from the $k_{T}$ factorization approach on the quarkonium production puzzles, Phys. Rev. D66 (114003) 2006.

[7] V.A.Khoze, A.D.Martin, M.G.Ryskin, and W.J.Stirling, Inelastic J/ $\psi$ and $\Upsilon$ hadroproduction, Eur. J. Phys. C39 (163) 2005.

[8] A. Abulencia, et al.,Polarization of $J / \psi$ and $\psi(2 S)$ mesons produced in p anti-p collisions at $s^{* *}(1 / 2)$ = 1.96-TeV. ,Phys. Rev. Lett. 99 (132001) 2007.

[9] V.M. Abazov, et al., Measurement of the polarization of the Upsilon(1S) and Upsilon(2S) states in $p$ anti-p collisions at $s^{* *}(1 / 2)=1.96-T e V, P$ hys. Rev. Lett.101 (182004) 2008.

[10] J. Thome and J. Russ, A Measurement of Upsilon(1S) Polarization, CDF Public Note 9966 (1) 2009. 